\title{
THE ROLE OF SUSTAINABLE ARCHITECTURE IN VALUABLE HISTORICAL DISTRICTS OF TEHRAN (A CASE STUDY OF SUSTAINABLE RESIDENTIAL DEVELOPMENT IN SANGLAJ DISTRICT)
}

\author{
Mahdi Makari Faraji \\ PhD of Architecture, Department of Architecture, Dubai, Islamic Azad University, Dubai, United Arab \\ Emirates \\ Eskandar Mokhtari Taleghani \\ $\mathrm{PhD}$ of Architecture, Department of Architecture, Dubai, Islamic Azad University, Dubai, United Arab \\ Emirates \\ Mahdi Makari Faraji \\ MSc of Architecture, Department of Architecture, Dubai, Islamic Azad University, Dubai, United Arab \\ Emirates \\ Mahdi.f60@gmail.com
}

\begin{abstract}
In this paper we study the role sustainable architecture in valuable historical districts of sangalj in city of Tehran. Different aspects of architecture were studied in this paper. Results show that contemporary architecture has an important effect on the face of building in Tehran. Furthermore the modern architecture in valuable city textures is one of challengeable issues in modern architecture and renovation in Iran. The old textures of Tehran are faced to severe structural erosion and social decline because of backwardness from city evolution and development. Social problems that are the result of these erosions cause the escape of local residents and replaced with other citizens that not believe there as their living place.
\end{abstract}

Keywords: City evolution, sustainable architecture, sangalj, Social problems.

\section{INTRODUCTION}

Sustainable architecture as well as other important issues in architecture involves the particular principles which this alone includes the factors such as storage of resources, designing to return to the life cycle and design the sustainable architecture for the humans with particular strategies. It could be stated that in modernism and modernization eras, Iranian architecture may not played its distinctive role-consequently no changes have been manifested in Iranian architecture in these eras (Arnason, 1376pp5). The application of contemporary architecture in valuable urban textures is a question which it is used in most of the countries in worldwide. For this, previous experiences show that whether the contemporary architecture has faced the indifferences or has it faced the negligence?" Iran is one the countries which joined to the committee to support the cultural and natural heritage. It seems that the noticeable samples may be observed in contemporary architecture in Iran which all these samples could be well-known in worldwide sample in near future. Green construction-Sustainable building refers to a structure and using process that is environmentally responsible throughout a building's life-cycle: from sitting to design, construction, operation, maintenance, renovation, and demolition. Actually the perception of an environment by the inhabitants in a neighborhood affects the relation of them with the environment and the level of their satisfaction (Sylvie Faucheux, 2007). 
It is argued that the characteristics of the person and their relations with each other are important factors determining residential satisfaction. Satisfaction is considered as one of the main characteristics of residential environmental assessment and so it was defined- "high quality environment". Thus, it is believed that satisfaction is the very general characteristic to assess perception of environmental quality (Apliard, 1382).

Bauerhas announced in his study that urban sustainable architecture could lead to the advancement of life quality in urban structure by which the advancement of environmental quality observes (Bauer, 2010).

Ghobadian stated in a research that individuality in selecting a place for living has become common in developing countries, in such a way that everyone with a better income choose better places for living as well by which quality of life and more convenience would be realized. Environmental quality can define as a larger concept of "quality of life"; combining of basis qualities as health, safety with aspects of welfare and grace (Ghobadian, 2009).

Pankstated in his research that a city constructed with sustainable architecture has been taken into account the objectives of sustainable architecture by which aspects of quality and especially satisfaction of residents involving livability, living quality, living environment, quality of place, residential-perception and- satisfaction, the evaluation of the residential and living environment, quality of life and sustainability would be realized (Pank, 2002).

Pellihad come up with the point that today, the quality of architecture has become a concerning for architects which it is observed with the titles "sustainable architecture" and "Green architecture" (Pelli, 1994).

In present paper, the architecture of Sangalaj hall in Tehran has been perused.

\section{QUESTIONS}

Has the Sustainable architecture applied in historical places regarding the social and cultural relations and also climatic changes?

Have the adjacent areas in Tehran located in historical places regarding the sustainable architecture?

Sustainable architecture has to be applied in the well-known historical places which with regard to social and cultural relations and also climatic changes, this would be possible.

With regard to sustainable architecture, we could design and construct the adjacent areas in Tehran locating in historical places.

\section{METHODOLOGY}

According to the issue of a research in any academic research, selection of an appropriate method is a very important issue by which a key concept relevant to a discussion of research methodology is that of validity. By a method in the research, the aims of the research would be realized. Therefore, defining scientific method means representation of fundamental guidelines which is important in any research. There are various methods in social science including field and documentary method, which in present paper the documentary method has been used for data collection in the overview of literature section (Campbell, 2001).

It has to be taken into consideration that any research has to be started from a point which this brings about several questions in researcher's mind by which the hypotheses would be represented, so that providing study and searching to prepare responses for the questions mentioned in the research and in order to get the conclusion would be the important duty undertaken by a researcher, which through the data collection this would be possible. The stage for data collection is the beginning of a process in which 
the researcher collect the field and library studies, and after that through the inferential method, his categories and analyzes them the assessment of hypotheses would be realized. It could be said that after these stages, the responses would be provided.

\section{Case study research method}

This research is in fact of the quantitative type and is performed through phenomenology and case study in which taking measures to reply the research questions is possible, and also in present paper an operational sample is created to present the preliminary plan .

The research type is practical, and the strategy of this research is the inferential. In this method, through tangible methods the data is collected, analyzed and developed then. This research is performed through the descriptive approach including evaluation of viewpoints and opinions. The data are collected through the methods of questionnaire, interview or observance. In this process, information and data are purified through both theoretical and experimental methods in order to draw out the necessary results. Through the descriptive and comparative methods, the obtained data could be observed by two following factors are essential.

\section{The Method for Data Collection}

This method has been used for data collection in the field of overview of literature, which within studying the books, paper and researches accomplished by other authors and surfing the net, the data collection would be provided. Along this, the library method and asking help from specialists who accomplished helpful studies in this field were taken into account as the first stage in this research. For instance through the study accomplished by Sassi, it could be concluded that within the increase of problems in modern world, sustainable structures were taken into consideration in developed countries. While in developing countries like Iran, the methods was implemented to reduce the social, cultural and environmental matters, which in this case economic consequences would be realized? The topic "sustainable architecture" is not a serious matter in our country (Iran), but a new method "green building" introduced in Iran which many people are persuaded to apply this method in their life. The way to implement and apply the method "Green building" has been involved in the strategies represented for sustainable architecture (Sassy, 2008).

Selecting an appropriate method is from the important issues in which the accuracy in the process of selecting could provide the desirable results. Hence, defining a scientific method means defining a fundamental guidelines presented in any research project. There are various methods in a research which we could mention documentary and field study in this case, so that in overview of literature in this research the documentary method was used. Along this, through having a conversation with Atabak Naderi on the basis of sustainable architecture of sangalaj theater , says, applying development architecture on sangalaj is a project which from one years ago taken into consideration. According to the studies accomplished on it, the mayor demanded the reports to do the first observations which subsequently the architecture development on Sangalaj Theater would be realized. In this relation, the artist Atabak Naderi stated that municipality has still being worked on this project which in this case more observations are needed by which the last outlook would be presented. The new director of the Sangalaj Theater is inviting a number of veterans to come on stage to revitalize the old hall in downtown Tehran, which was once the haunt of the lovers of traditional Iranian plays, says , We are currently negotiating with artists who played a key role in the development of the Sangalaj Theater in its early days, and some of them have agreed to perform .We also intend to showcase a clear reflection of the hall's activities, and to show that it is moving in line with other national Iranian theaters, of which traditional theaters are one subdivision. Naderi was attempted to apply the development architecture on Sangalaj Theater by which on the basis of presentation of the reports about this project, he stated that the exact time is not definite. For this, the mayor has to state his final report .As a matter of fact, we are in hurry for getting results from this project but there is not time limitation for this. In this relation, he stated that the projects accomplished over the years to develop the Sangalaj Theater architecture were the primary projects; in 
fact more development projects would be represented for this. Accordingly, the studies accomplished over the years were mainly prepared to show the geographical position of this theater .Furthermore on the basis of establishment of theater, Atabak Naderi stated that this geographical area is the old texture located in Tehran bringing about cultural messages .It could be stated that existence of a museum for theater lovers is desirable; Meanwhile, There are documents in museums which are needed to be kept safe. The documents in the theater belonged to the past periods shown the necessity for the establishment of museum by which the development architecture for Sangalaj Theater has been recommended. It has been stated that Naderi has also talked to Jafar Vali, Jamshid Layeq, Jamshid Mashayekhi, Davud Rashidi, and Bahram Beizaii for this which all of them referred to municipality.

\section{Data Collection Method}

Data collection is performed through the following methods:

1-Interview through purposeful sampling

2-Observation

3-Documentary

4-Audio and Visual

5-Acquired from the virtual space

And the accessible resource for data collection includes:

First Hand Resources

\section{Second Hand Resources}

Different books written by internal and foreign authors, different journals relevant with architecture and building construction, using internet to search various resources are other means for data collection.

In first section, interviews are performed with different participants including the designers of sustainable developments, constructers and even in some cases it will be performed with the users of sustainable development. Filling out the questionnaire is also another part which is used for asking opinions.

\section{Data Analysis}

Descriptive Analysis includes Summarization and classification of data collection through producing and regulating the tables and graphs

We have collected the data in this research using the descriptive analysis method which we categorized them using the indices of descriptive statistics, thereafter we summarized them.

Take permission to exploit from the historical places which through providing the finances from the public and private sectors, the historical places could be renovated.

\section{RESULTS AND DISCUSSION}

Sangalaj is one of the four neighborhoods of primarily old core of Tehran. It is a neighborhood survived from old time with it primarily structures and has to remain alive in a new big metropolis. This neighborhood is facing with many challenges which are emerged because of mismatch of what objectives over them the neighborhood has formed and what needs now people with new lifestyles expect. Now neighborhoods like this in the literature of planning in Iran are recognized as problematic areas. On the other hand, it was attempted to renovate the architecture of Sangalaj by new orientations of planning in Iran which is shown its new buildings, spaces, streets, infrastructures and etc based on approaches trying 
to make environments for new people. This planning maybe affected by modern styles has willing to make it most far from old parts and old styles. According to the modern lifestyles, the evaluation of residential environment quality in these different districts can be indicator of values and qualities of each environment.

Currently, Architects play very important role to use sustainable architecture, this is due to the fact that $50 \%$ of energy consumption in all over the world is going to be dissipated Construction coverage involve all the construction components separating outside and inside of the construction. Designing the exterior walls construction techniques and components.

solar rays , temperature, humidity and wind, noise, fire, insect and animals, solar rays , temperature , humidity and wind, noise, fire, insect and animals, second factor, total energy consumption of the green building are The factors for The choice of materials. The choice of materials depend largely upon their properties relative to environmental requirements and their strength. And methods is needed to allow them to be used more efficiently and effectively.

Iranian architecture or Persian architecture is the architecture of contemporary Iran .It has a continuous history from the past up to now Iranian architecture displays great variety, both structural and aesthetic, developing gradually and coherently out of earlier traditions and experience.

The sustainable architecture was more observed in traditional constructions; Sustainable architecture is a general term that describes environmentally conscious design techniques in the field of architecture.

Using the sun's energy is a better choice which is not harmful for individual's health. Design with accurate layout helps the citizens to use the solar energy by which the desirable light would be also

\section{Neighborhoods and districts of Tehran:}

The city of Tehran is divided into 22 municipal districts, each with its own administrative centers. Within these 22 districts, Tehran contains the following major neighborhoods:

Tehran is bound in the north by the massive Alborz mountain range that is stopping the flow of the humid Caspian wind. As a result, thermal inversion that traps Tehran's polluted air is frequently observed. The lack of humidity and clouds makes Tehran a very sunny city.

Case study in this paper are Sanglaj, the neighborhood inside Tehran. Sanglaj is one of the four neighborhoods of primarily old core of Tehran. It is a neighborhood survived from old time with it primarily structures and has to remain alive in a new big metropolis. This neighborhood is facing with many challenges which are emerged because of mismatch of what objectives over them the neighborhood has formed and what needs now people with new lifestyles expect. Now neighborhoods like this in the literature of planning in Iran are recognized as problematic areas.

It has to be taken into account that residential environment quality evaluation in different districts can be indicator of values and qualities of each environment.

Sangalaj quarter since Agha Mohammad khan sat on the throne until decease of Mohammad shah; Sangalaj quarter was located in the northwest and west of the city and was nonresidential area until Naserddin shah era. In this era, some of the nobles of the city and court created gardens this quarter at the end of kingship of Naserddin shah, the uncultivated lands were granted to the people in order that they construct house in there and lack of housing be compensated. Sangalaj quarter was located in the west of old Tehran and it has been mentioned as one of the oldest quarters and undoubtedly, its name is related to the era before Safavieh. This name is seemingly old and ancient. There had been other places in the ancient time that their name had been similar to this name. Sangalaj quarter which had been in Transoxiana that has been in the mountain slope and there had been a ruby mine in the mountain; this 
name with this description has been mentioned in Hodoodolalam, but it is not clear that whether it has any relationship with Sangalaj quarter in Tehran regarding derivation. Some of the scholars state that the origin word is Sang Raj and relate it to partitioning water with stones. During second era, from erection of first rampart until Tehran became capital in 1169 Jalali calendar, Tehran city was in the medieval style. The bazaar including various caravansaries, arcades, and passageways and the main passageways linked the quarters to each other; for instance, Gozar Emamzadeh Yahya and Mahaleh Oudlajan end up in Darvazeh Qazvin. The framework of Tehran based on linkage among centers of quarters and city center via linking spaces means main passageway and open spaces. There is little structures such as Gozar Mahaleh Emamzadeh Yahya, Sabzeh Meydan, and some parts of Grand bazaar as public spaces are considered as vestige of mentioned eras. The limitation of Tehran or Hesar shah Tahmasebi end up into Amirkabir Ave, and Imam Khomeini in the north, Rey Ave in east, Molavi Ave in south and Vahdat Islami Ave in the west. With regard to 5th era, from the formation of city and development of the city toward north, it could be stated that the most critical transformations with framework during this era is the conceptual formation called as public building(Administrative, Bank, police, university, school, and etc) which transformed the medieval style of Tehran to modern style. Upon the space of Citadel, Mashq square had been destroyed, and the new ministries have been built as well as instead of a part of Sangalaj which was destroyed, then park shah was built.

From 1929 to 1934 Tehran grows twice in a period of five years. In 1937 the first urban development plan for Tehran was designed. It was highly influenced by the modern movement and international style with an imposing a new road system, wide, tree-lined streets intersecting at right angles upon the old fabric, a Cartesian grid, functional zoning and production of public space. The Royal compound was fragmented. Its buildings, although redeveloped, were to be replaced by a new government quarter, mainly the Ministries of Justice and Finance. The surviving buildings were transformed for other uses like the Golestan Palace, which became a museum (Isesko, 2006). The Sangalaj neighbourhood was bulldozed for construction of the stock exchange building -- which was never implemented due to the Second World War. The ruins remained deserted until 1950, and according to the decision of the Council, eventually turned into the Central Park of the city (Apliard, 2003). This plan was the first intervention plan in the historic inner city. The plan considered the city as a construction site instead of a living creature which not only led to the scattering of the urban structure but also the socio-cultural institutions of the inhabitants. About the plan of Sangalaj quarter in 1896, it could be stated that two gates connected to this quarter in outside of the city, Qazvin gat that Shapoor square was constructed there in later and Mohammadieh gate that Edam square replaced it later. There is no shrine in this quarter except seyed Naserddin while there is 13 shrines in other quarters. Perhaps the lack of shrine has been the reason that its lands was barren and undeveloped since the ancient time until the beginnings of Qajar era. While there had been a main water resource named "Sarab Pakhshkon", the people resided in this quarter due to population increase since the beginning of kingship of Naserddinshah and created some small quarters most of which became renown with the title deriving from name of the people residing in there such as 1Ghoorkhaneh Kohneh quarter, 2- Bajmanlooha quarter, 3-Dabaghkhaneh quarter,4- Chaleh Hesar quarter, 5- Hematabad quarter, 6-Pachenar quarter, 7- Qomiha quarter, 8- Aramaneh quarter, 9-Baq Khosrokhan quarter, 10- Torkamanha quarter, 11- Darvazeno quarter; it seems that the oldest place of Sangalaj is the places around Seyednasreddin which it is located between sangalaj quarter and bazaar. About the Passageways existing in sangalaj quarter, it could be stated that there had been some passageways in sangalaj quarter including 1-Sandoghdar small bazaar passageway,2- Filmkhaneh passageway,3Darkhoongah passageway that it's name and location of some of them still remains like Darkhoongah passageway that derivation of the word is not clear.

Sangalaj based on plan 1930, capital, governed by Abdolghaffar Hamedani:

In the changes made after transformation of location of Tehran walls, the most changes were made in sangalaj quarter and the reason was that the main part of these lands were purchased by Mirza Yousef 
Mosofilmamalek in his chancellery and took measures to develop it. These lands were extended from today's Rahahn square to the neighborhood of DEh vanak. Contrasting plan of 1930 with the previous plan shows that important changes had been made in the west of Tehran in the term between presenting these two plans.

Gates in sangalaj quarter

There are four gates including 1- Khaniabad,2- Gomrok, 3-Ghazvin, 4- Bagh shah connected to sangalaj quarter with the outside the city. The two gates, Gomrok and Bagh shah had been recently constructed. Khaniabad gate was along the same Mohammadieh gate which had been constructed in Mohammadshah era. Ghazvin gate had been constructed in the past.

Quarters in sangalaj quarter

The quarters including 1- Ghoorkhaneh kohneh(in the north of sangalaj quarter and west of Arg), 2Bajmanlooha(in the north of Tekieh Abpakhshkon),3-Dbaghkhaneh(in the south of Abpakhshkon), 4Argiha(in the northeast of section of Abpakhshkon or jadid), 5- Haj Sheikh hadi(in the east of Abpakhshkon or jadid), 6- Chaleh Hesar(in the Galoobandak passageway), 7-Hematabad (in the east of Ghomiha quarter), 8-Ghomiha(in the north of Moayarolmamalek),9- Darvaezeh No (in the south of Imamzadeh Zeid and Aramaneh alley), 10- out of Darvazeh Ghazvin "Ghazvin gate"(in the south of Aramaneh street and the section of sangalaj quarter or Jadid), 11- out of Darvazeh No (in the sout of Moaryaromamalek street in the section of sangalaj quarter), 12-Jadid(in the west of out of Darvazeh No in the section of sangalaj quarter or jadid) could be seen in the plan.

Streets in sangalaj quarter

Streets in sangalaj quarter were as follows: 1- Jalilabad,2-Amirabad(from east to west),3Markizkhaneh(along Amirabad street),4-Haj Sheikh hadi(from north to south),5- Nosratddoleh,6Ghavamoddoleh,7-Haj Sheikh Hadi, 8- Bagh Jannat,9- Amirieh(from north to south),10-Darvazeh Ghazvin,11- Nosratddoleh Dovom(second),12-Darvazeh no, 13- Ghapoogh, 14- Darvazeh Gomrok,15Bagh Moayarolmolk.

Original Tehran had four residential quarters. These residential quarters were clustered around the axis of bazaar- as the backbone of the city and the focus of life - were called: Sangalaj, Chal-meidan, Bazaar and Oud-lajan. Sangalaj was razed and converted into City Park. Chal-meidan is no longer distinct -- it is a shadow of its former self. And, while Bazaar no longer serves as a residential neighborhood, commerce continues to thrive during the day. Most unfortunate of all, Oud-lajan now ranks as the most neglected of the four. Once a wealthy residential neighborhood, Oud-lajan is now mainly a staging area for commerce in Bazaar. For this and other reasons, which I will soon make clear, Oud-lajan is moribund. It has become a symbol of decay and neglect for all residents of Tehran, and has gained a reputation as a hotspot for crime. Today, Oud-lajan is placed in Tehran's 12th municipal district. In present paper, the architecture of Sangalaj hall in Tehran has been perused. Sangalaj is one of the four neighborhoods of primarily old core of Tehran. It is a neighborhood survived from old time with it primarily structures and has to remain alive in a new big metropolis. This neighborhood is facing with many challenges which are emerged because of mismatch of what objectives over them the neighborhood has formed and what needs now people with new lifestyles expect. Now neighborhoods like this in the literature of planning in Iran are recognized as problematic areas. On the other hand, it was attempted to renovate the architecture of Sangalaj by new orientations of planning in Iran which is shown its new buildings, spaces, streets, infrastructures and etc based on approaches trying to make environments for new people. This planning maybe affected by modern styles has willing to make it most far from old parts and old styles. According to the modern lifestyles, the evaluation of residential environment quality in these different districts can be indicator of values and qualities of each environment. 


\section{CONCLUSION}

Through the observation of the present paper, it has been concluded that, however, the architecture in Iran has been changed in perspective of economic result and materialism in the procedure of life in the modernity elements. Hence, to preserve the resources for the future generation- we have to act in accordance with the acts in the worldwide. The application of new energies in the construction and making the architecture coordinated with ecology could help us in the optimization and control of energy consumption by which the pollution of environment would be avoided. This leads to the increase of life quality and convenience in the human's life in their mind and physics. Today, despite representation of sustainability issues, practically all the principles were being used by previous generation- in fact in the past all the architects were good at utilizing the natural resources and energies. The exploitation from nature realized in different levels and was taken from various resources. The observation about the strategies for traditional architecture with the objective of consistency with environment could be used for the today's architecture. Today, within the advancement of technology- the methods could be adapted with the requirements by which the optimized usage of natural energies would be realized. In this relation, it has to be stated that architects and project developers can get past the idea and use solar panels in their designs. For most architects, the visual and aesthetic aspects are the most important. They try to create a spatial object with lines, shapes, colors and texture. These are the challenges for the architect within the customer's program of requirements. But they do not immediately think of using a solar panel as an interesting building material. There is still much to be achieved here. In addition to the design and art/science definition described above, architectural conservation also refers to issues of identification, policy, regulation, and advocacy associated with the entirety of the cultural and built environment. This broader scope recognizes that society has mechanisms to identify and value historic cultural resources, create laws to protect these resources, and develop policies and management plans for interpretation, protection, and education. Typically this process operates as a specialized aspect of a society's planning system, and its practitioners are termed built or historic environment conservation professionals. Architectural conservation describes the process through which the material, historical, and design integrity of mankind's built heritage are prolonged through carefully planned interventions. The individual engaged in this pursuit is known as an architectural conservator. Decisions of when and how to engage in an intervention are critical to the ultimate conservation of the immovable object. Ultimately, the decision is value based: a combination of artistic, contextual, and informational values is normally considered. In some cases, a decision to not intervene may be the most appropriate choice.

On the basis of construction materials, it has to be stated that the materials involve the diversity, and the construction field has been expanded through the modern engineering instrumentations. Exploitation of the construction materials while choosing them and using the pertinent construction method to implement the construction domain would lead to the high quality of construction- in fact the environmental issues help for the better construction. Applying the modern construction methods help the designer to access the objectives of sustainable development which through this, the environmental issues would be also helpful in the construction industry. Surely, there are other consequences gained in present paper as following:

The concepts and the principles of architecture follow the culture of a territory.

The formation of architecture follows the environmental and philosophical characteristics by which the factor "time" has been mentioned as the fundamental principle for the formation of the new spaces.

According to the sustainable principle in any phenomenon, the principles could be preserved and be used as the fundamental concepts.

To keep the culture and art of a territory, getting inspired of the previous forms is not important which being inspired of the previous architecture design could be mentioned as the necessity in designing the innovative art and outlook

Submit Date: 11.06.2016, Acceptance Date: 22.07.2016, DOI NO: 10.7456/1060AGSE/068 Copyright (C) The Turkish Online Journal of Design, Art and Communication 
It could be stated that architecture could transit the meaning, not the form

Paying attention to the architecture does not mean to imitate the realistic philosophy of the foreign territory. In the world of communication, it could be stated that technology, information and knowledge all belong to the human civilization which the exploitation from these three technologies, information and knowledge would be probable, and this is possible only through conserving the philosophical and cultural principles. Imitation does not mean in a superior art" architecture" which it could be stated that trust could not be put on the art" architecture" without innovation, dynamism, reality and creativity. There is not form for the concept of culture which in order to advance the art" architecture" in a territory, the innovation would be effective to access the concepts in this relation.

The formation of architecture involves semiologic and symbolic forms which in this relation it has to be stated that the construction with the combined elements of the architecture could not move out of the cultural cognitions. Experiencing the modern art" architecture" which is the stoppage of the relationship between environment, tradition, and history is the necessary symbol of a failed experience.

The symbol could be observed in all the phenomena. Through the philosophy, literature, art, knowledge and etc the symbols could be taken out.

The architecture involves two fundamental cultural and academic principles which by the passage of time if one of the principles annihilates so that the consequences would not be obtained. In the field of architecture, creativity and the innovation are from the fundamental principles which through these both principles the dominancy on the art would be realized.

As a matter of fact, in the procedure for the formation of architecture- various factors like ecology, consumption, performance, the materials, human force and the economy of the construction would be effective for the physic of the construction. Also, in the procedure for the impacts of the material, physical, environmental and ecological factors, the form and design of the construction have to be involved of all the values of a society. The culture attained in a construction is influenced of the factors for the human science and the innovative art allocated for the culture of the territory. Culture Architecture's purpose is to empower individuals and organizations to achieve whole new levels of performance and effect lasting cultural change. The individuals and organizations are educated to dramatically improve productivity resulting in increased satisfaction and profit on the basis of architecture. Culture Architecture's value proposition is simple: We provide leading-edge personal and professional development tools and training; delivered in a modern, easy to access structure. Our cost effective methodology is highly impactful and provides a high return on investment. The training is designed to engage and empower every person as a leader who can train and empower others, regardless of their history, position or current circumstances.

As observed over the years, after several years simultaneously with City Theater reconstruction, Sangalaj Theater was reconstructed as well. At first, the reconstruction was only limited to replacing the chairs and floor, whereas no reconstruction on this theater was accomplished up to now. For this, Atabak Naderi, director of Sangalaj Theater request for reconstruction of this theater from the industrial projects department in ministry of Islamic culture and guidance, by which the agreement was issued. The initial cost for reconstruction of Sangalaj Theater was low, but post approving this project the cost was increased very much. According to the consultations accomplished in centers for the performing arts, it has been attempted to allocate the Sangalaj Theater only for traditional performances ; Due to the fact that this kind of performing only attracts little fans, so that, Sangalaj Theater would not be like the past with many fans .

Lack of theater halls could be mentioned as one of the main problems in the theater domain in the country; allocation of Sangalaj hall with its interesting background is a brilliant phenomenon in the 
country. Due to gaining so many capabilities post reconstruction, and also based on technical system observing in Sangalaj hall, annually various performances could be organized in this hall .But there is a point to say which it is highlighted that due to organizing traditional performances in this hall, it might not be taken into account . So many people believe that due to geographical position of this hall, it might not be taken into account as well.For this, the performances organized in this theater might not be potential and attractive, by which no addressee refer to this reconstruction of Sangalaj theater - a performance played by Akbar Abdi , Iranian Actor attracted many people and gained success , which this performance accounted as the most sale performance organized in this theater. This phenomenon shows that, however, Sangalaj may be located in an unfavorable geographical location, there is a contradictory so as it could be said that organizing various performances in this theater could attract many people .This is possible in a way that due to the location of Sagalaj in vicinity of subway, many problems may be resolved .It has to be noticed that through performing art center 's support and the support by artists of theater and performing various performances, Sangalaj theater hall regained it 's popularity among individuals, in such a way that the distribution of advertisements in perspective of Sangalaj could be lead to the success of theater in making money .

Hence, helpful strategies to advertise Sangalaj theater hall could be effective in this way .The other effective point in making Sangalaj theater hall prominent is the type of executing this hall. A young, skilled and sympathetic director could control and undertake the management of this theater hall in a best way by which, many positive messages would be resulted .Atabak Naderi is the sample of a good manager who referred to this theater hall in reconstruction period .Naderi's referral to this theater hall lead to manifestation of the points which these points were latent.

Naderi put the attempt to clear the latent points. theater community has to be thankful from these directors who try the most to keep the theater community dynamic .Fortunately, according to the various programs provided for the upcoming theater programs, so that these programs could be taken into account only while the directors in the theater domain believe all the facts in this relation.

\section{REFERENCES}

Arnason, H (1997), the history of modern art, Islamieh Mostafa, Tehran.

Apliard, Donald (2003) streests can led to the cities dead, translate: Novin Tavalaei, Abadi mag, NO: 39, page $73 \_83$.

Campbell, N.S. and S.Stankovic, 2001. Wind Energy for the Built Environment: Project WEB. ImperialCollege, Mecal Applied Mechanice and the University of Stuttgart. London. Isesko (1385) report of isesko function in Tehran during 1993-2005, Tehran.

Michael Bauer, Peter Mösle, Michael Schwarz.2010. Green Building: Guidebook for Sustainable Architecture.

Pank, W., H. Giradet, G. Cox. 2002. Tall buildings and sustainability. Report. Faber Maunsell for the Corporation of London.

Pelli, C. and Crosbie, M.J. 1994. Cesar Pelli: Selected and Current Works. Images Publishing.

Sassi, P. (2006). Strategies for sustainable architecture. Taylor \& Francis.

Ghobadian ,Vahid.2009. Sustainable Traditional Buildings of Iran(A climatic analysis).universal company publisher \& distributor.

Sylvie Faucheux, Martin O'Connor, Jan van der Straaten.2007.Sustainable development: concepts, rationalities, and strategies. 